\title{
Is Drawing Video Game Characters in an Hour of Code Activity a Waste of Time?
}

\author{
Ashok Basawapatna \\ SUNY College at Old Westbury \\ Old Westbury, New York, USA \\ basawapatnaa@oldwestbury.edu \\ Josiane Manera \\ FHNW Switzerland \\ Windisch, Switzerland \\ josiane.manera@fhnw.ch
}

\author{
Alexander Repenning \\ FHNW Switzerland \\ Windisch, Switzerland \\ alexander.repenning@fhnw.ch \\ Nora Escherle \\ FHNW Switzerland \\ Windisch, Switzerland \\ nora.escherle@fhnw.ch
}

\author{
Mark Savignano \\ Minnesota State University, Mankato \\ Mankato, Minnesota, USA \\ mark.savignano@mnsu.edu \\ Lorenzo Repenning \\ AgentSheets Inc. \\ Boulder, Colorado, USA \\ lorenzo.repenning@gmail.com
}

\begin{abstract}
Broadening participation in computer science necessitates balancing motivational and educational concerns. Without fully understanding potential trade-offs, Hour of Code-like tutorials may actually backfire by initially attracting students to participate, but gradually reinforcing the notion that programming is hard and boring. Previously, we analyzed and compared two Hour of Code tutorials: a tutorial that walks students through the creation and programming of a 3D-Frogger game, including the drawing of their own $3 \mathrm{D}$ characters, to a programming puzzle where students solve discrete coding challenges. Using an analysis based on retention, the comparison indicated higher levels of perseverance in the game creation activity. However, does the ability to draw characters really motivate students to program? Conflicting theories of positive and negative effects of drawing onto perseverance include that drawing might increase levels of participant ownership or that drawing may just be wasting time better spent programming, especially in the time constrained Hour of Code context. To gain insight, this study uses draw times of over 8,000 projects from a game creation Hour of Code activity in 2016. Initial results indicate that higher average draw time per character corresponds to increased program lengths and students with the highest average draw times per character continued to program beyond the end of the activity
\end{abstract}

\section{CCS CONCEPTS}

- Applied computing $\rightarrow$ Interactive learning environments; Computer-managed instruction;

\section{KEYWORDS}

Computer science education; online education; evaluation; retention; Flow; cyberlearning; CS Ed Week; Hour of Code; creativity; drawing; art

Permission to make digital or hard copies of all or part of this work for personal or classroom use is granted without fee provided that copies are not made or distributed for profit or commercial advantage and that copies bear this notice and the full citation on the first page. Copyrights for components of this work owned by others than ACM must be honored. Abstracting with credit is permitted. To copy otherwise, or republish, to post on servers or to redistribute to lists, requires prior specific permission and/or a fee. Request permissions from permissions@acm.org.

ITiCSE'18, July 2-4, 2018, Larnaca, Cyprus

(C) 2018 Association for Computing Machinery.

ACM ISBN 978-1-4503-5707-4/18/07 ..\$15.00

https://doi.org/10.1145/3197091.3197136

\section{ACM Reference Format:}

Ashok Basawapatna, Alexander Repenning, Mark Savignano, Josiane Manera, Nora Escherle, and Lorenzo Repenning. 2018. Is Drawing Video Game Characters in an Hour of Code Activity a Waste of Time? In Proceedings of 23rd Annual ACM Conference on Innovation and Technology in Computer Science Education (ITiCSE'18). ACM, New York, NY, USA, 6 pages. https://doi.org/10.1145/3197091.3197136

\section{INTRODUCTION}

There are many encouraging developments in the ongoing effort to expose students to programming activities and computational thinking. One example is Computer Science Education Week (CS Ed Week) containing the Hour of Code which challenges students across the world to spend an hour writing their first program [18]. Students, many with little to no prior coding experience, pick from an assortment of highly scaffolded online activities offering a gentleslope introduction to programming [10]. The Hour of Code has boasted participation numbers in the hundreds of millions $[5,18]$.

Giant participation numbers underscore the critical importance of evaluating these activities to ensure maximum engagement by participants; failing to do so risks reinforcing the preconceived notion that programming is "hard and boring" [5, 7]. Even now, five years after the Hour of Code launched, there has been woefully little research and analysis on the aspects of online Hour of Code tutorials that are potentially motivational or de-motivational to students.

Our greatest hope is that every Hour of Code activity is a "cliffhanger" leaving students highly motivated to continue after activity completion. However, students could go through the activity, enjoy it, and never return to coding. The ephemeral nature of the Hour of Code increases the possibility of it becoming a mere one-time enrichment activity like a "trip to the zoo." In a post activity survey, such as [5], or in Code.org's self-released impact study [11], this would show up in the form of positive responses and more positive student dispositions towards computer science, and activity designers would be none the wiser. In the worst-case scenario, the activity could be an "are we there yet" experience with students struggling to get through the hour, or bored throughout the hour, resulting in loss of all interest to ever program in the future.

To shed light onto potentially de-motivational and motivational aspects of these activities, we developed a method to analyze Hour of Code activities called the Retention of Flow. Retention of Flow 
uses participant retention, which follows a negative exponential starting at $100 \%$, at the beginning of the activity, and declining as people drop out of the activity, and compares it to a Markov chain model of retention to identify points of activity improvement [15] The Retention of Flow conjecture relates Csikszentmihalyi's idea of Flow [4] to student activity retention as follows. As participants complete one step of an activity, they gain skill that they can use to complete subsequent activity steps. One might aim to design an activity such that if the participant can complete the first step, the participant can complete all subsequent steps. This implies no drastic increase in challenge between steps as related to the current participant skill level. The Retention of Flow conjecture states that without any marked increase in challenges between steps, the continuation probabilities at each step of the activity are approximately identical, yielding a predicted negative exponential retention curve. Furthermore, points in the activity where the activity retention data greatly diverges from this predicted curve, are potential candidates for activity improvement, as they may indicate a mismatch of challenge and participant skill [15].

Further research extended the Retention of Flow to create a modified A-B testing method developed for evaluating Hour of Code activities [2]. Employing this methodology over two Hour of Code activity implementations in a two-year time-span, allowed for the identification of a problematic activity instruction, through the Retention of Flow methodology described above, and subsequent improvement of this activity via a simple activity modification. This improvement led to a significant number of participants avoiding the identified activity pitfall in their Hour of Code project.

As part of this previous research, we compared the retention of an Hour of Code activity, which walks students through the creation and programming of their game, to a programming game activity consisting of discrete puzzles $[12,14]$. We noticed that the retention of the activity wherein students created a $3 \mathrm{D}$ version of the video game Frogger was higher by the end of an hour than that of the programming puzzle, consisting of discrete levels where users employ code to guide an Angry Bird character towards a maze goal [12]. We further speculated that a higher retention after the first hour, along with evidence of participants continuing to program after the activity is complete, implies a cliff-hanger activity. This motivates a key question of analysis relevant to all Hour of Code activities: What are the key characteristics of Hour of Code activities that yield higher participant retention throughout the activity, programming past the first hour of the activity, and programming after activity completion?

One major difference between the two analyzed activities is that the game creation activity results in a unique artifact that the student creates and keeps. The process of designing and creating this artifact includes the drawing of one's own in-game characters. Furthermore, students can continue adding to the game using the programming environment after the activity is complete. Much research has been done into how ownership motivates students in educational computer science such as programming and storytelling [9], creation of educational video games [1], and constructing software to learn math [8]. In the Scratch environment, the ability to personalize a programmed game through art has been identified as a major factor in making learning activities that are meaningful to novice students [17]. In our own research, we have found

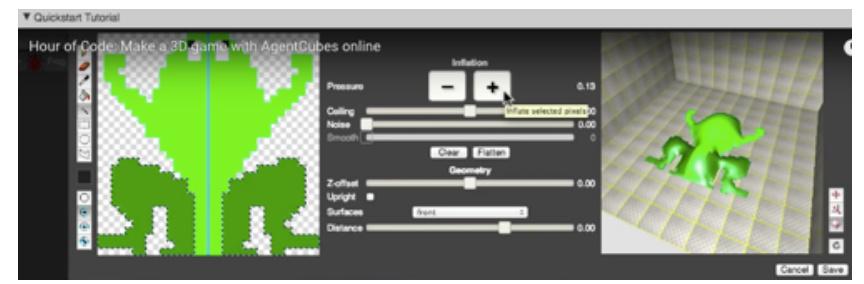

Figure 1: The Inflatable Icon Editor in AgentCubes online. On the left is the 2D character and on the right is the resulting 3D figure with hind legs inflated.

ownership and creativity to be important for student engagement. One student summed it up perfectly- when asked why he still enjoyed the game he created even though the graphics were not as sophisticated as Xbox games, he stated, "I like it because I made it" [16].

However, the Hour of Code context is an inherently time constrained endeavor. Students have one hour to code something meaningful, which makes drawing seem like a dispensable time waster. In previous professional developments, novice teachers have frequently worried about students dedicating too much time to drawing when doing an in-class programming unit. Veteran teachers conjecture that the ability to draw their own characters increases class enthusiasm, and broadens participation, but at the same time, will often advise novice teachers during training to stop students after five minutes of drawing. Specifically, they recommend telling the students to carry on programming, and reminding them that they will have time to continue drawing their characters later.

Given these two schools of thought, as part of continued efforts to improve Hour of Code activities, we decided to investigate if and how the opportunity to draw effects the programming of an activity. The hope is that Hour of Code activities can use this analysis to inform activity design strategies increasing student engagement, motivation, and retention.

\section{METHODOLOGY}

Data was collected from 8,614 games from the 2016 AgentCubes Online Hour of Code exercise where students create a 3D version of Frogger $[6,15]$. The activity is online browser based and students program their game using drag and drop conditions and actions to create behavior rules for their game characters, also called "agents." The instructional activity video, which is embedded in the programming environment, walks students through the step by step creation of their Frogger game in six different languages. A more in-depth description of the activity can be found in [6]. Notably, for this paper's purposes, the activity guides students through how to draw, in three dimensions, five of the eleven video game characters they need for Frogger. For the remaining six characters, students are instructed to choose from a set of pre-made character shapes.

Figure 1 depicts the Inflatable Icon editor in the Frogger 3D Hour of Code activity in AgentCubes online. The editor allows students to create a drawing in two dimensions and then inflate parts of the two-dimensional drawing into a three dimensional shape $[13,16]$.

Table 1 lists the five agents students draw throughout the activity along with the corresponding lines of code measurement (LOC), 
Table 1: The agents participants are instructed to draw at given program lengths (lines of code) and the time of drawing instruction given for each agent in the activity tutorial video. The new character drawn at the beginning of each tutorial section is in red. Does not include characters that participants create but are not directed to draw

\begin{tabular}{lll}
\hline $\begin{array}{l}\text { Lines of code } \\
\text { or } \\
\text { program length }\end{array}$ & $\begin{array}{l}\text { Characters drawn } \\
\text { at each stage with } \\
\text { new one in red }\end{array}$ & $\begin{array}{l}\text { Tutorial } \\
\text { draw time } \\
\text { (min) }\end{array}$ \\
\hline 0 to 20 & Frog & 4.28 \\
21 to 32 & Frog Road & 0.867 \\
33 to 48 & Frog, Road, Tunnel & 1.533 \\
50 to 52 & Frog, Road, Tunnel, Log & 1.0833 \\
53 to 78 & Frog, Road, Tunnel, Log, Bridge & 1.55 \\
\hline
\end{tabular}

also called program length, for when those agents exist in the activity along with the time the tutorial video dedicates to drawing each agent.

Table 1 is organized such that each part starts with the drawing of the new character before any lines of code are written. For example, at the beginning of the tutorial section that starts at 33 lines of code and ends at 48 lines of code, participants are first instructed to draw the tunnel. Based on these calculations, if a participant's program reaches 35 lines of code, we can take their total draw time and divide it by three (for the three agents they've drawn: frog, road, and tunnel) to arrive at the average time a participant spent drawing. This analysis assumes that the participant follows the activity and does not draw additional agents. Manually analyzing 100 projects, distributed evenly among each line of code range in Table 1, shows that this assumption was correct for $81 \%$ of the projects sampled. The validity of this assumption will further be covered in the discussion.

Readers familiar with previous research on the Retention of Flow $[6,14,15]$ will notice the lines of code numbers at similar points of the program are different-this is due to a change in the conversion from the visual language to its XML representation, from which lines of code are determined [15]. Though the lines of code calculation changed, the number itself is not as important as is the ability to associate lines of code to a certain point in the activity which allows for the identification of which step in the activity a participant dropped out. For the data presented in this paper, if a participant follows the activity tutorial exactly, they end up with 78 lines of code. However, post activity instruction, students can continue adding to their game in the open ended environment.

The activity tutorial video instructs the drawing of all 5 characters in Table 1 from start to finish. The final column of Table 1 displays the amount of time the tutorial video spends drawing each agent in real time. The average time per agent shown in Table 1 the video narrator spends drawing is 1 minute and 52 seconds, with most of the time being spent on the frog (4 minutes and 17 seconds).

In the first section of the tutorial video, the narrator draws the frog, programs the frog to move, and programs a win-state for the game, which consists of a goal the frog must get to. This first section lasts about 15 minutes, with approximately one third of that time spent on drawing the frog. In terms of program length, the first part of the activity amounts to 20 lines of code as measured in this study. This first section is typically what a student will finish in an hour of class-time and has been noted in hundreds of classroom observations over the last 9 years [14, 15].

The nature of retention necessarily means that there will be less students at the end of the activity, and beyond, than at the beginning. This has the downside of allowing a fewer number of students to bias our analysis of average draw times per agent vs. program length. To avoid this, we calculated the estimated sample size for a simple regression to obtain a power of 0.95 with an alpha error of 0.05 across all the groups sampled, arriving at a sample size of 420 . This equates to 70 randomly sampled projects from each group (code range) in Table 1 along with 70 projects that continued beyond the end of the activity (lines of code>78) for a total of 420 projects sampled.

By analyzing the draw times of these games, we hope to establish an understanding of connections between the use of design activities and programming in Hour of Code type tutorials.

\section{RESULTS}

The results section is split up into three parts: a regression analysis, an average draw time retention analysis, and an analysis of the first part of the activity. The discussion section that follows will talk in-depth about these results and conclusions.

1. Regression Analysis. Using the sampling methodology described in the previous section, a simple linear regression was calculated to predict lines of code based on draw time per character. Results of this regression are as follows $(\mathrm{R} 2=0.048, \mathrm{~F}(1,420)=20.93, \mathrm{p}=0.01)$ with $(\beta=0.034, \mathrm{p}<0.05)$. The small $\mathrm{R} 2$ value indicates $4.8 \%$ of the variation in lines of code can be explained by draw time per agent. The p-value indicates that this minor positive correlation is significant, showing a relationship between greater draw times and increased program length.

2. Average Draw Time Per Agent Retention Analysis. Students are guided through the creation of five agents in the activity. Figure 2 shows the average draw time per agent for every project greater than or equal to a given program length. For example, averaging the draw time per agent of all games with lines of code indicating they got to the end of the activity and beyond ( $>=78$ lines of code) indicates an average draw time per agent of 5.67 minutes. This figure was created using the sampling methodology outlined above to ensure equal numbers of students in each group. This figure shows that students who get to the end of the activity and beyond have higher draw times per agent. Also of note, the average draw time per agent stays level, at a little over 4 minutes per agent, until program length 50 where it begins to increase. Finally, a polynomial trend line of order 2 is overlaid on the data and proves to be a good predictor of the average draw time for all the projects past a given line of code value with an r-squared of 0.98 .

The apparent inflection point at program length 50 in Figure 2 motivated a comparison of draw times between the group of students with lines of code less than 50 and those with lines of code greater than 50 to determine if there existed a significant difference between these two groups. We chose to compare each 


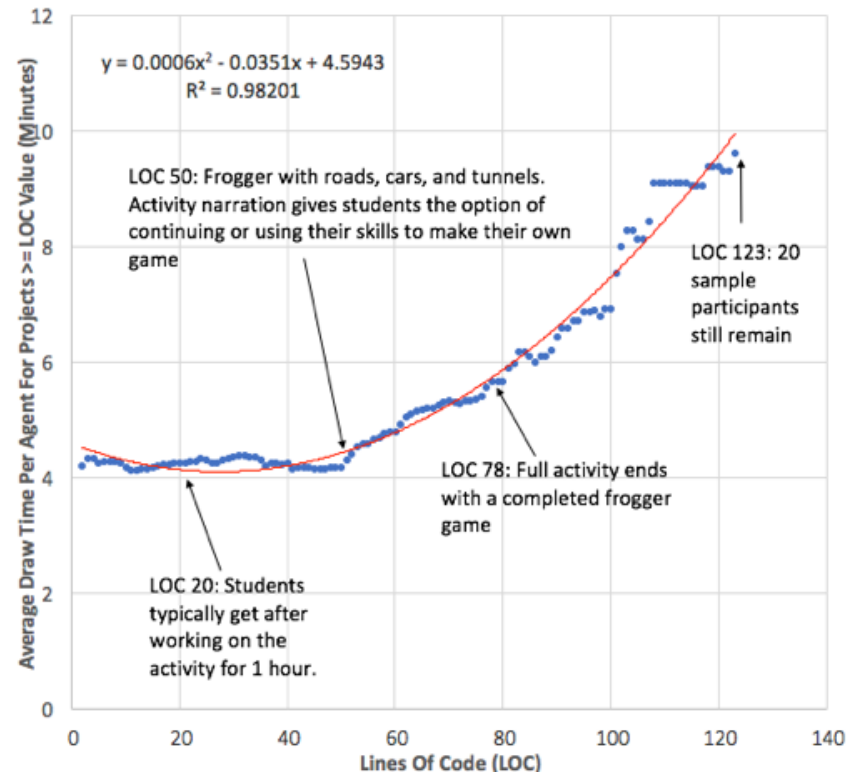

Figure 2: The Average Draw Time Per Agent for all the projects with lines of code greater or equal to a given program length

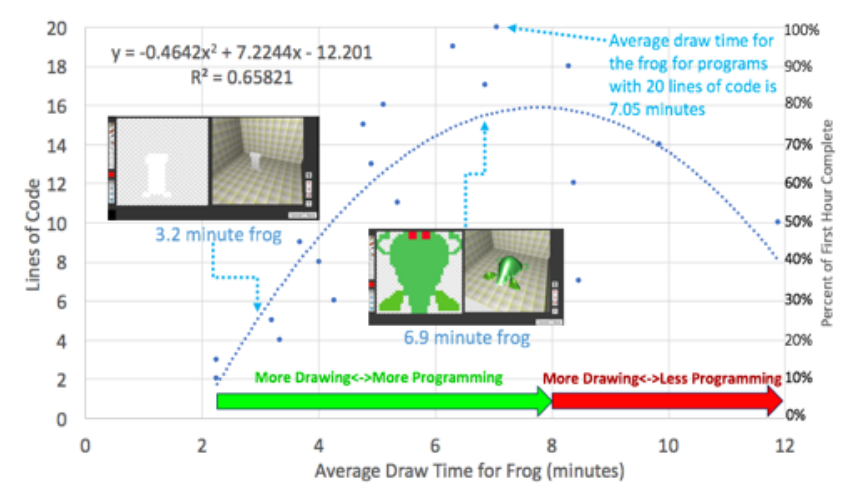

Figure 3: Lines of Code vs. Average Draw Time in minutes for projects 20 lines of code or less with a best fit order 2 polynomial overlaid.

group through an ANOVA and resampled from the full participant data for this analysis. We estimated a sample group of 420 would help us achieve a power of 0.95 with a level of significance at 0.05 . After running the analysis, the p-value was at 0.033 , which was lower than the level of significance of 0.05 . This suggests that there is indeed a significant difference between these two groups.

3. Lines of Code vs. Average Draw Time For the First Part of Activity.

As stated above, in an hour, students typically create the frog, program it to move, and program a win state for the game. If all of this is done correctly, their programs will have a length of 20 lines of code. About a third of the first part of the activity, which takes 15 minutes on the video, is spent drawing the frog. Figure 3 shows an analysis of just the projects that have 20 lines of code or less. At each program length, from 2 to 20 (the frog agent with an empty rule is 2 lines of code), the average draw time of all the projects with that particular program length are calculated and plotted (lines of code vs. average draw time). Unlike the previous analysis comparing different groups, this plot averages all the projects, as opposed to sampling, from the first group in Table 1. This data shows that, starting from the left and going right, as draw times increase, the program length increases, with a sweet spot of students who finish or get close to finishing part 1 spending around 6-8 minutes drawing. After that point, increased draw times are not necessarily associated with longer programs.

\section{DISCUSSION}

The discussion section is organized into three parts. The first part is an in-depth discussion of the results with section numbers corresponding to the section numbers in results. The second part of the discussion presents the implications of the results to this and other Hour of Code activities, and the third part discusses the shortcomings of this study.

\subsection{Discussion of Results}

1. Regression Analysis. The results from the linear regression analysis predicting lines of code based on draw time per character begin to show that drawing and coding are not zero sum, with projects containing both increased average draw times and larger program lengths. The weak positive but significant correlation between lines of code and average draw time per agent indicates that, on the whole, spending more time on drawing may not be the hindrance to programming that many teachers feared.

2. Average Draw Time Per Agent Retention Results. Figure 2 looks at the average draw times per agent for every project with lines of code greater or equal to a given line of code value. Looking at this it is clear that students who finished the whole activity, and went beyond, typically had larger draw times per agent. For example, the average draw time for students who wrote 100 lines of code or more was around 7 minutes per agent. Of course, students who went past the end of the activity may have created more agents on their own, inflating the draw time per agent number. However, the more important point is that students who were so engaged with the activity to keep on programming also spent significant amounts of time drawing. The ascending section of the graph starting at program length 50 implies that, consistently, at each subsequent program length, students on the lower side of average draw time per agent dropped out more than students on the higher side. To put this another way, students who went went deeper into the activity, as measured by lines of code, tended to spend more time drawing than those who did not.

There are two conclusions we can surmise from this. First, if we are looking for indicators of what makes a cliff-hanger activity, this graph shows that for the students who tend to get to the end of the activity and beyond, the ability to draw their own characters was important. Students either spent more initial time drawing and/or continued to draw on their own, even when not explicitly instructed to by the activity, yielding a higher draw time average. Second, eliminating the ability to draw in an effort to dedicate maximum 
time on programming might have unintended consequences in terms of stifling these high achieving students.

Figure 2 also points to a more nuanced view of drawing and program length. Specifically, until program length 50 we see no drastic changes in what looks to be a fairly horizontal line with average draw time per agent of around 4 minutes. After 50 however, the curve starts to steadily increase implying that students with lower average draw time per agent values start dropping out of the activity. The ANOVA results strongly suggest that program length 50 is an inflection point by indicating a significant difference between the pre 50 lines of code group and the post 50 lines of code group based on draw times. A follow up qualitative study is necessary to identify the specific differences between these two groups, but analyzing the activity at that point allows us to speculate why this might have occurred. At line 50 the average student has programmed a basic Frogger game. Around this point the video's instructional narration says the following: "So now you've learned 3 essential computational thinking patterns: collision...,generation..., and absorption... With these skills you can build sophisticated games of your own or you can continue to follow along with me as I add more creatures and objects to this $3 D$ world."

The narrator essentially gives students the option of stopping. It seems reasonable that less engaged students might use this as an invitation to quit the activity (altogether or possibly to work on another game), while more engaged students would continue. However, as to why exactly these students are engaged or not engaged is an open question. For example, it could be that students who drop out simply do not enjoy drawing and those who continue do. Or students who draw more have a greater attachment to their game. Either way, Figure 2 could have shown that students with higher average draw times drop out earlier yielding a descending curve. This is clearly not the case as the data indicates increased drawing does not necessarily correspond to reduced program length.

3. First Part of Activity Analysis. Figure 3 shows that as draw times for the frog increase, so do program lengths, up to a point. However, after that point, the draw times increase and program lengths decrease. An interpretation for low draw times being associated with less lines of code could be that low draw times indicates a student who is less engaged in the activity as a whole. High draw times could be detrimental in that the student is so engaged in drawing $3 \mathrm{D}$ agents that, by the time they move towards programming, the class is over. However, it could also mean that the student is having trouble with the drawing tools and cannot create as quickly as they would like.

\subsection{Implications}

There are two major implications to this study. The first is that in the time constrained context of the Hour of Code, spending a significant amount of time drawing may not be detrimental to the programming part of the activity and may actually enhance it. In Figure 3 we see that in the first part of the activity, spending more time drawing the frog agent actually corresponds to more lines of code, up to a point. Furthermore, in Figure 2 we see for students who completed the activity and went above and beyond, drawing did not seem to be an obstacle, but rather, something they actively did.
Students do not typically learn how to code in one hour. The best outcome is that by activity's end, students' interests are piqued and they are motivated to continue coding. As mentioned above, we want to know what aspects of Hour of Code activities make it an "are we there yet" activity vs. a "cliff-hanger activity." Our data shows that the students who were motivated to continue coding past the hour mark to the end of the activity and beyond were also motivated to draw as they continued coding or perhaps, code as they continued drawing. This indicates that the presence of drawing has helped make this a cliff-hanger activity for many students.

The second implication is the opportunity to modify this activity to better support students as they create their agents. For instance, we might employ the Zones of Proximal Flow construct [3], wherein students who are in a state of anxiety are guided back into the Flow [4] through scaffolding. Combined with qualitative research as to why student draw times are lower, the results of this paper could be used to identify which students are in the Flow based on metrics such as average draw time per agent. For example, what if we could use the best fit curve in Figure 2 to accurately predict the program length a student is on pace to get to based on their average draw time per agent? This could lead to teacher or activity intervention, similar to Piech et. al [12], in an effort to reengage struggling students and guide them back into the Flow.

\subsection{Study Shortcomings}

Exclusively keeping track of the total draw time forces the deduction of draw time per agent through use of Table 1. However, if students deviate from the activity, which they are welcome to do by the activity narration, the validity of these measures will be off for that particular student. As mentioned above, a manual analysis of 100 programs indicated that around $81 \%$ of the submissions followed Table 1. Better instrumentation might include a draw time counter for each individual agent as well as a session counter indicating when someone returns to an agent to modify its appearance. Furthermore, a more qualitative study of the programs with high draw times might yield a better understanding of what students actually accomplish when their draw times are so high.

In addition to finer grained and more accurate draw time per agent measurements, there are a few other issues with this study. The first is the uncertainty regarding the extent to which drawing was actually a motivator for students. To truly understand this, we must do a qualitative factor analysis study to decipher what motivates and engages students as they go through this Hour of Code activity. A survey into motivations/engagement or an interview into student perceptions and what they enjoy could help paint a more accurate picture of the quantitative data. It would also be interesting to do a qualitative study to better understand sub groups of students drawing appeals to and if there are any groups of students who are repelled by the initial act of drawing in an Hour of Code activity.

Another issue has to do with server difficulties during the Hour of Code 2016. At the beginning of the week, the participation rate was so high that it overloaded the server leading to many students who could not attempt this particular Hour of Code activity and possibly included many students who may have started but did not have a chance to finish because of the server issues. This is bound 
to modify the retention data. In previous research we looked at technical challenges showing up as barrier for continuation in these activities [15]. For example, someone who encounters technical challenges might have a low draw time per agent and drop out at a low program length. In this study, that student would incorrectly be interpreted as having little motivation or engagement in the activity.

Related to the idea of outside factors, we previously identified practical challenges to student retention [15]. For example, after an hour the student might stop because class is over. To complete the entire Frogger activity takes a classroom setting that dedicates multiple hours to the activity, or a student who works outside of class to finish her/his game. However, that might not be possible, for example, if a student does not have access to a computer outside of school. This student would appear as someone who spent a long time drawing the frog, did not program many lines of code, but to this study, looks like a person who spent too much time drawing and thus, did not get to code. A brief online questionnaire after the activity could provide a better clue as to who these students are and give us indications of what they thought of the activity including practical barriers to returning if any.

\section{CONCLUSION}

This study builds upon previous Retention of Flow research to analyze how drawing relates to student programming progress in an Hour of Code activity. Students do not learn coding in an hour, and thus the judgement as to of whether an Hour of Code activity is successful or not hinges on whether the student continues to code after activity completion. This research indicates that drawing is not a waste of time, even in the time limited context of the Hour of Code, and in fact, could be a major factor in creating a "cliff-hanger" activity for some students. This provides an important initial data point to better understand how drawing and coding can coexist and indicates that drawing might actually ameliorate the coding activity as students draw and code past activities' end and beyond. Hour of Code tutorials have the power to motivate hundreds of millions of students in computational thinking. However, by the same token, they also have the capacity to confirm stereotypes of programming to be "hard and boring." It is imperative to continue criticially analyzing these activities, disseminating their strengths and weaknesses, and improve these activities in order to ensure an engaging and motivating first exposure to computer science for the greatest numbers of students.

\section{ACKNOWLEDGMENTS}

Data used with permission from AgentSheets Inc. Inflatable Icon is patented under US Patent No. 7,630,580. This work is supported by the Hasler Foundation, the Swiss National Science Foundation under Grant CRAGP2_158545, and the National Science Foundation under Grant Numbers 0833612, 1345523, and 0848962. Any opinions, findings, and conclusions or recommendations expressed in this material are those of the authors and do not necessarily reflect the views of these organizations.

\section{REFERENCES}

[1] Tiffany Barnes, Heather Richter, Eve Powell, Amanda Chaffin, and Alex Godwin. 2007. Game2Learn: Building CS1 Learning Games for Retention. In Proceedings of the 12th Annual SIGCSE Conference on Innovation and Technology in Computer Science Education (ITiCSE '07). ACM, New York, NY, USA, 121-125. https://doi. org $/ 10.1145 / 1268784.1268821$

[2] Ashok Basawapatna and Alexander Repenning. 2017. Employing Retention of Flow to Improve Online Tutorials. In Proceedings of the 2017 ACM SIGCSE Technical Symposium on Computer Science Education (SIGCSE '17). ACM, New York, NY, USA, 63-68. https://doi.org/10.1145/3017680.3017799

[3] Ashok R. Basawapatna, Alexander Repenning, Kyu Han Koh, and Hilarie Nickerson. 2013. The Zones of Proximal Flow: Guiding Students Through a Space of Computational Thinking Skills and Challenges. In Proceedings of the Ninth Annual International ACM Conference on International Computing Education Research (ICER '13). ACM, New York, NY, USA, 67-74. https://doi.org/10.1145/2493394. 2493404

[4] Mihaly Csikszentmihalyi. 1997. Finding flow: The psychology of engagement with everyday life. Basic Books.

[5] Jie Du, Hayden Wimmer, and Roy Rada. 2016. "Hour of Code": Can It Change Students' Attitudes toward Programming? fournal of Information Technology Education: Innovations in Practice 15 (2016), 52-73.

[6] Nora A Escherle, Silvia I Ramirez-Ramirez, Ashok R Basawapatna, Dorit Assaf, Alexander Repenning, Carmine Maiello, Yasko Ch Endo, and Juan A NolazcoFlores. 2016. Piloting computer science education week in Mexico. In Proceedings of the 47th ACM Technical Symposium on Computing Science Education. ACM, 431-436.

[7] Google CS Ed Research group et al. 2014. Women who choose computer sciencewhat really matters: The critical role of encouragement and exposure. Technical Report. Technical report, Google. Retrieved from http://static. googleusercontent. com/media.

[8] Idit Harel and Seymour Papert. 1990. Software design as a learning environment. Interactive learning environments 1, 1 (1990), 1-32.

[9] Caitlin Kelleher and Randy Pausch. 2007. Using Storytelling to Motivate Programming. Commun. ACM 50, 7 (July 2007), 58-64. https://doi.org/10.1145/ 1272516.1272540

[10] Deepak Kumar. 2014. Digital Playgrounds for Early Computing Education. ACM Inroads 5, 1 (March 2014), 20-21. https://doi.org/10.1145/2568195.2568200

[11] Rachel S. Phillips and Benjamin PC Brooks. 2017. The Hour of Code: Impact on Attitudes Towards and Self-Efficacy with Computer Science. (2017). Retrieved April 1, 2018 from http://code.org/files/HourOfCodeImpactStudy_January.pdf

[12] Chris Piech, Mehran Sahami, Jonathan Huang, and Leonidas Guibas. 2015. Autonomously Generating Hints by Inferring Problem Solving Policies. In Proceedings of the Second (2015) ACM Conference on Learning Scale (LS '15). ACM, New York, NY, USA, 195-204. https://doi.org/10.1145/2724660.2724668

[13] Alexander Repenning. 2005. Inflatable icons: Diffusion-based interactive extrusion of 2d images into 3d models. Fournal of Graphics Tools 10, 1 (2005), $1-15$.

[14] Alexander Repenning and Ashok Basawapatna. 2016. Drops and Kinks: Modeling the Retention of Flow for Hour of Code Style Tutorials. In Proceedings of the 11th Workshop in Primary and Secondary Computing Education (WiPSCE '16). ACM, New York, NY, USA, 76-79. https://doi.org/10.1145/2978249.2978260

[15] Alexander Repenning, Ashok Basawapatna, Dorit Assaf, Carmine Maiello, and Nora Escherle. 2016. Retention of Flow: Evaluating a Computer Science Education Week Activity. In Proceedings of the 47th ACM Technical Symposium on Computing Science Education (SIGCSE '16). ACM, New York, NY, USA, 633-638. https://doi. org/10.1145/2839509.2844597

[16] Alexander Repenning, David C Webb, Catharine Brand, Fred Gluck, Ryan Grover, Susan Miller, Hilarie Nickerson, and Muyang Song. 2014. Beyond minecraft: Facilitating computational thinking through modeling and programming in $3 \mathrm{~d}$. IEEE Computer Graphics and Applications 34, 3 (2014), 68-71.

[17] Mitchel Resnick, John Maloney, Andrés Monroy-Hernández, Natalie Rusk, Evelyn Eastmond, Karen Brennan, Amon Millner, Eric Rosenbaum, Jay Silver, Brian Silverman, and Yasmin Kafai. 2009. Scratch: Programming for All. Commun. ACM 52, 11 (Nov. 2009), 60-67. https://doi.org/10.1145/1592761.1592779

[18] Cameron Wilson. 2015. Hour of Code-a Record Year for Computer Science. ACM Inroads 6, 1 (Feb. 2015), 22-22. https://doi.org/10.1145/2723168 